\title{
INTERCORRÊNCIAS CLÍNICAS E OBSTÉTRICAS VIVENCIADAS POR MULHERES NO PRÉ-NATAL
}

\author{
Rafaella da Silva Calegari ${ }^{1}$, Helga Geremias Gouveia ${ }^{2}$, Annelise de Carvalho Gonçalves ${ }^{3}$
}

RESUMO: Objetivou-se conhecer as intercorrências clínicas e obstétricas em mulheres no pré-natal e verificar a concordância do relato da mulher com registros da carteira de pré-natal associadas às intercorrências com o tipo de parto. Trata-se de um estudo transversal, em um Hospital Universitário, com 361 puérperas, de fevereiro a abril de 2013. Na análise, procedeu-se à estatística descritiva e aplicação do teste qui-quadrado. Observou-se que $67,9 \%$ das mulheres tiveram alguma intercorrência, a mais frequente foi a infecção do trato urinário, porém, houve coincidência entre registro na carteira pré-natal e relato nos casos de síndrome hipertensiva, infecção do trato urinário, trabalho de parto prematuro e diabetes gestacional. Não houve associação estatisticamente significante entre as intercorrências e tipo de parto. Esses achados podem possibilitar o planejamento de ações que direcionem medidas de promoção de saúde.

DESCRITORES: Saúde da mulher; Obstetrícia; Cuidado pré-natal.

\section{CLINICAL AND OBSTETRIC COMPLICATIONS EXPERIENCED BY WOMEN IN PRENATAL CARE}

\begin{abstract}
The objective was to get to know the clinical and obstetric problems women experience in prenatal care and to verify whether the women's reports agreed with records on the prenatal care history, associating the problems with the type of birth. A cross-sectional study was undertaken at a University Hospital, involving 361 puerperal women between February and April 2013. In the analysis, descriptive statistics and the chi-squared test were applied. It was observed that $67.9 \%$ of the women experienced some problem. The most frequent was urinary tract infection, but the records in the prenatal history agreed with the reports in cases of hypertensive syndrome, urinary tract infection, premature labor and gestational diabetes. No statistically significant association was found between the problems and the type of birth. These findings can permit the planning of actions to guide health promotion measures.
\end{abstract}

DESCRIPTORS: Woman's health; Obstetrics; Prenatal care.

\section{COMPLICACIONES CLÍNICAS Y OBSTÉTRICAS VIVIDAS POR MUJERES EN EL PRENATAL}

RESUMEN: La finalidad fue conocer las complicaciones clínicas y obstétricas en mujeres en el prenatal y verificar la concordancia del relato de la mujer con registros en el archivo de prenatal, asociando las complicaciones con el tipo de parto. Se trata de un estudio trasversal, en un Hospital Universitario, con 361 puérperas, de febrero a abril del 2013. En el análisis, fueron aplicadas la estadística descriptiva y la pruebaji-cuadrado. Fue observado que el 67,9\% de las mujeres vivió alguna complicación. La más frecuente fue la infección del tracto urinario, pero coincidieron los registros en el archivo prenatal y relatos en los casos de síndrome hipertensiva, infección del tracto urinario, trabajo de parto prematuro y diabetes gestacional. No fue encontrada asociación estadísticamente significante entre las complicaciones y el tipo de parto. Esos hallazgos pueden posibilitar la planificación de acciones que dirigen medidas de promoción de salud.

DESCRIPTORES: Salud de la mujer; Obstetricia; Cuidado prenatal.

`Enfermeira. Universidade Federal do Rio Grande do Sul. Porto Alegre, RS, Brasil.

Enfermeira. Doutora em Ciências da Saúde na área de Saúde Materna e Perinatal. Docente de Enfermagem da Universidade Federal do Rio Grande do Sul. Porto Alegre, RS, Brasil.

${ }^{3}$ Enfermeira. Doutora em Saúde Pública. Docente de Enfermagem da Universidade Federal do Rio Grande do Sul. Porto Alegre, RS, Brasil.

Autor Correspondente:

Rafaella da Silva Calegari

Universidade Federal do Rio Grande do Sul

Av. Panamericana, 257/303 - 91050-001 - Porto Alegre, RS, Brasil

E-mail: calegarirafaella@gmail.com
Recebido: 02/01/2016 Finalizado: 06/04/2016 


\section{INTRODUÇÃO}

Toda mulher que está em período gestacional necessita e tem direito a uma assistência pré-natal de qualidade, importante tanto para a saúde materna quanto para a saúde do feto ${ }^{(1)}$. Essa assistência de qualidade e diferenciada, de acordo com o risco gestacional, promove uma melhoria na saúde de ambos e mostra-se como uma prevenção para possíveis agravos ${ }^{(2)}$.

A gestação é um fenômeno fisiológico que envolve mudanças físicas, sociais e emocionais que podem implicar em riscos para a mulher e para o feto. Há uma parcela de gestantes que, por características particulares, apresentam maior probabilidade de evolução desfavorável da gestação, essa são as chamadas gestantes de alto risco ${ }^{(3)}$. Tanto em gestações de alto risco quanto nas de risco habitual, pode haver a ocorrência de alterações no curso fisiológico da gestação, o que denominamos de intercorrências obstétricas, em que há alteração da evolução e do andamento do processo gestacional fisiológico. Muitas vezes, o desfecho dessa gestação, ou seja, o tipo de parto, pode ser influenciado por essa intercorrência ${ }^{(4)}$.

Na maioria dos casos, o parto normal é a maneira mais segura e saudável para o nascimento do bebê. Ainda são feitas muitas cesarianas desnecessárias. Ressalta-se que estas devem ser realizadas somente quando há algum risco para a mãe e/ou para o bebê( ${ }^{(1)}$.

Em 2013, (56,6\%) ${ }^{(5)}$ dos partos realizados no Brasil foram cesarianas. A recomendação da Organização Mundial da Saúde (OMS) é que a taxa de cesárea seja no máximo $15 \%{ }^{(6)}$. Os casos de gestantes com hipertensão, por exemplo, estão acarretando um aumento na incidência de cesáreas, pois são distúrbios considerados graves durante a gestação(7).

Entre as causas de morte materna, as que prevalecem no Brasil são a hipertensão, a hemorragia e a infecção puerperal. No Rio Grande do Sul, a primeira causa é a hipertensão ${ }^{(8)}$.

Segundo a OMS, "mais de meio milhão de mulheres no mundo morrem por causas maternas a cada ano"(9:1). As mortes maternas e também fetais ocorrem, muitas vezes, por problemas relacionados à prevenção, ou seja, causas que poderiam ter sido evitadas durante as consultas de pré-natal ${ }^{(9)}$.

Entre os oito Objetivos de Desenvolvimento do Milênio (ODM), estabelecidos pela Organização das Nações Unidas (ONU), o quinto objetivo propôs a redução da mortalidade materna, entre 1990 e 2015, em três quartos. Relatório, apresentado em 2014, apontou que entre 1990 e 2011, o Brasil reduziu 55\% dos óbitos, passou dos 141 para 64 óbitos por 100 mil nascidos vivos. Contudo, a razão de óbitos maternos ainda permanece acima da meta estipulada para 2015 de 35 óbitos por 100 mil nascidos vivos, mostrando que o Brasil enfrenta desafios para alcançar a redução da mortalidade materna na magnitude preconizada ${ }^{(10)}$.

Destaca-se a necessidade de uma assistência obstétrica humanizada e de um elo forte entre as mulheres e o serviço de saúde. Observa-se que, em alguns casos, o diagnóstico da intercorrência clínica e/ou obstétrica no pré-natal é tardio, podendo culminar em condições materno-fetais desfavoráveis ${ }^{(11)}$. As intercorrências poderiam ser evitáveis durante o pré-natal com medidas como serviços de educação e esclarecimento e locais de referência bem equipados, de fácil acesso a essas mulheres ${ }^{(11)}$, bem como acompanhamento adequado que favoreça o diagnóstico e tratamento precoce. É conveniente que todas as mulheres, mesmo antes de engravidar, tenham acesso aos serviços de saúde e às informações cabíveis para uma boa condução da gestação mediante a utilização de meios de comunicação, visitas domiciliares e atividades educativas ${ }^{(3)}$.

Levando em consideração a repercussão das intercorrências para a mulher, feto ou recém-nascido, acredita-se ser relevante conhecer as intercorrências clínicas e obstétricas frequentes vivenciadas por mulheres durante o período do pré-natal, verificar a concordância do relato da mulher e o registro na carteira pré-natal e associar as intercorrências com o tipo de parto. Entende-se que esses dados podem contribuir para o planejamento e implementação de ações e medidas de promoção da saúde para essas mulheres. Além disso, a enfermagem poderá intensificar medidas de educação em saúde e estratégias de prevenção desses acometimentos, norteando a assistência às gestantes atendidas na instituição estudada. 
Este estudo é uma subanálise da pesquisa denominada "Fatores associados à realização de cesariana em Hospital Universitário". Trata-se de um estudo quantitativo de corte transversal. Foi desenvolvido na Unidade de Internação Obstétrica de um Hospital Universitário de Porto Alegre no período de fevereiro a abril de 2013. Para o cálculo do tamanho amostral, foi considerado o número total de partos ocorridos em 2011, que foi de 00203714, e a taxa de cesariana que foi 37,4\% (1392 cesarianas). Considerando a referida taxa de cesarianas, com uma margem de erro absoluta de $5 \%$ e nível de confiança de $95 \%$, compuseram a amostra 361 puérperas. Para realização desse cálculo foi utilizado o Software Win Pepi versão 11.43.

Foram incluídas na amostra mulheres cujo parto foi realizado no Centro Obstétrico da referida instituição, seja parto vaginal ou cesárea (eletiva ou de urgência). Foram excluídas as que não tiveram condições psicológicas de responder as questões, os casos de óbito fetal, fetos com peso inferior a 500 gramas e/ou com idade gestacional inferior a 22 semanas.

Os dados primários foram obtidos na planilha de ocorrências da área restrita da unidade de centro obstétrico, de registros do prontuário físico e eletrônico materno, da carteira pré-natal e da entrevista estruturada. Procedeu-se a teste piloto, com a aplicação do instrumento de coleta de dados com 10 puérperas. Foram realizados ajustes e adaptações, também testados, até se obter a versão final do instrumento.

As variáveis de interesse para este estudo foram a caracterização da amostra, a história obstétrica, intercorrências na gestação, segundo as anotações da carteira pré-natal e/ou relatadas pela mulher, e o tipo de parto.

Para análise, foi utilizada estatística descritiva, e para a verificação da associação entre as variáveis do estudo, o teste de qui-quadrado. O software utilizado foi o SPSS versão 18. Por tratar-se de um projeto que envolve seres humanos, o projeto inicial foi submetido para avaliação ética ao Comitê de Ética em Pesquisa da Instituição, sendo aprovado com o registro número 120466 em 31 de janeiro de 2013. A pesquisa respeitou os termos da Resolução $n^{\circ} 466 / 12$ do Conselho Nacional de Saúde no que se refere aos aspectos éticos ${ }^{(12)}$.

\section{- RESULTADOS}

Entre as participantes do estudo, 245 mulheres (67,9\%) tiveram alguma intercorrência clínica e/ou obstétrica no pré-natal atual, e, dessas, 120 (49\%) tinham o registro de mais de uma intercorrência na carteira pré-natal (Tabela 1).

Entre as mulheres que apresentaram intercorrência(s) clínica(s) e/ou obstétrica(s) na gestação atual registrada(s) na carteira pré-natal, a maioria encontrava-se na faixa etária de 16 a 34 anos de idade $\mathrm{n}=207(84,5 \%)$, tinha mais de oito anos de estudo $\mathrm{n}=133(54,3 \%)$ e renda familiar de até dois salários mínimos $\mathrm{n}=161(65,7 \%)$. Em relação à história obstétrica, a maioria apresentava-se com duas ou mais gestações $n=153(62,4 \%)$, e, entre estas, cerca de metade era primípara $n=78$ (51\%) (Tabela 2).

A intercorrência mais frequente entre as mulheres, segundo o registro da carteira pré-natal, foi a infecção do trato urinário (104 casos). Quando analisada a relação das informações, verificou-se maior coincidência $(>90 \%)$ entre o registro da intercorrência na carteira pré-natal e o relatado pela mulher nos casos de síndrome hipertensiva, infecção do trato urinário, trabalho de parto prematuro e diabetes gestacional (Tabela 3).

No que se refere às principais intercorrências obstétricas apresentadas pelas mulheres, nenhuma teve associação com significância estatística com o tipo de parto - desfecho (Tabela 4). 
Tabela 1 - Distribuição das mulheres segundo o número de intercorrência(s) clínica(s) e/ou obstétrica(s) na gestação atual registrado na carteira pré-natal. Porto Alegre, RS, Brasil, 2015 ( $N=361)$

\begin{tabular}{lcc} 
Número de intercorrências & $\mathbf{N}$ & $\mathbf{\%}$ \\
\hline Nenhuma & 116 & 32,1 \\
\hline Uma intercorrência & 125 & 34,6 \\
\hline Duas intercorrências & 75 & 20,8 \\
\hline Três intercorrências & 29 & 8 \\
\hline Quatro intercorrências & 11 & 3 \\
\hline Cinco intercorrências & 3 & 0,8 \\
\hline Seis intercorrências & 1 & 0,3 \\
\hline Sete intercorrências & 1 & 0,3 \\
\hline Total & $\mathbf{3 6 1}$ & $\mathbf{1 0 0}$
\end{tabular}

Tabela 2 - Distribuição das mulheres que tiveram alguma intercorrência clínica e/ou obstétrica na gestação atual segundo idade, escolaridade, renda familiar, história obstétrica. Porto Alegre, RS, Brasil, $2015(\mathrm{~N}=245)$

\begin{tabular}{lcc} 
Variável & N & $\%$ \\
\hline Idade & 6 & 2,4 \\
\hline$<15$ anos & 207 & 84,5 \\
\hline 16 a 34 anos & 32 & 13,1 \\
\hline$>35$ anos & & \\
\hline Escolaridade & 66 & 26,9 \\
\hline$<8$ anos de estudo & 46 & 18,8 \\
\hline 8 anos de estudo & 133 & 54,3 \\
\hline$>8$ anos de estudo & & \\
\hline Renda familiar & 68 & 27,8 \\
\hline Até um salário mínimo & 93 & 37,9 \\
\hline Dois salários mínimos & 70 & 28,6 \\
\hline Três ou mais salários mínimos & 14 & 5,7 \\
\hline Não soube informar & & \\
\hline História obstétrica & 92 & 37,6 \\
\hline Primeira gestação & 153 & 62,4 \\
\hline Duas ou mais gestações & \\
\hline Parto anterior (N=153)† & 14 \\
\hline Nenhum parto anterior & 9,1 \\
\hline Primípara & 78 \\
\hline Multípara & 61 \\
\hline
\end{tabular}

tConsideradas somente as com duas ou mais gestações (história obstétrica).

Tabela 3 - Distribuição das mulheres que tiveram alguma intercorrência clínica e/ou obstétrica na gestação atual, segundo a concordância entre o registro na carteira pré-natal e relato das mulheres. Porto Alegre, RS, Brasil, 2015 $(\mathrm{N}=245)$

\begin{tabular}{|c|c|c|c|c|c|c|}
\hline \multicolumn{2}{|l|}{ Registro na carteira pré-natal } & \multicolumn{4}{|c|}{ Relato das mulheres } & \multirow{3}{*}{$\begin{array}{c}\text { Total } \\
71 \\
\end{array}$} \\
\hline & & \multirow{2}{*}{$\begin{array}{c}\text { Sim } \\
58\end{array}$} & \multirow{2}{*}{$\begin{array}{c}\text { \% } \\
81,7 \\
\end{array}$} & \multirow{2}{*}{$\frac{\text { Não }}{13}$} & \multirow{2}{*}{$\begin{array}{c}\% \\
18,3 \\
\end{array}$} & \\
\hline Amniorrexe prematuraf & Sim & & & & & \\
\hline & Não & 10 & 5,8 & 163 & 94,2 & 173 \\
\hline \multirow[t]{2}{*}{ Diabetes gestacional¥ } & Sim & 21 & 91,3 & 2 & 8,7 & 23 \\
\hline & Não & 4 & 1,8 & 217 & 98,2 & 221 \\
\hline \multirow[t]{2}{*}{ Estreptococo B $\ddagger$} & Sim & 24 & 58,5 & 17 & 41,5 & 41 \\
\hline & Não & 2 & 1 & 201 & 99 & 203 \\
\hline \multirow[t]{2}{*}{ Trabalho de parto prematuro $\ddagger$} & Sim & 23 & 92 & 2 & 8 & 25 \\
\hline & Não & 11 & 5 & 208 & 95 & 219 \\
\hline \multirow[t]{2}{*}{ Síndrome hipertensiva } & Sim & 37 & 94,9 & 2 & 5,1 & 39 \\
\hline & Não & 15 & 7,3 & 190 & 92,7 & 205 \\
\hline \multirow[t]{2}{*}{ Infecção do trato urinário } & Sim & 98 & 94,2 & 6 & 5,8 & 104 \\
\hline & Não & 23 & 16,3 & 118 & 83,7 & 141 \\
\hline
\end{tabular}

$\ddagger U m$ caso sem registro. 
Tabela 4 - Associação das intercorrências obstétricas com o tipo de parto. Porto Alegre, RS, Brasil, 2015 (N=245)

\begin{tabular}{|c|c|c|c|c|c|c|}
\hline \multirow[t]{2}{*}{ Intercorrência Obstétrica } & & \multicolumn{2}{|c|}{ Parto Vaginal } & \multicolumn{2}{|c|}{ Cesariana } & \multirow[t]{2}{*}{ p valor } \\
\hline & & $\mathbf{N}$ & $\%$ & $\mathbf{N}$ & $\%$ & \\
\hline \multirow[t]{2}{*}{ Amniorrexe prematura§ } & Sim & 48 & 67,6 & 23 & 32,4 & 0,78 \\
\hline & Não & 112 & 64,7 & 61 & 35,3 & \\
\hline \multirow[t]{2}{*}{ Diabetes Gestacional§ } & Sim & 12 & 52,2 & 11 & 47,8 & 0,234 \\
\hline & Não & 148 & 67 & 73 & 33 & \\
\hline \multirow[t]{2}{*}{ Estreptococo B§ } & Sim & 26 & 63,4 & 15 & 36,3 & 0,89 \\
\hline & Não & 134 & 66 & 69 & 34 & \\
\hline \multirow[t]{2}{*}{ Síndrome hipertensiva§ } & Sim & 21 & 53,8 & 18 & 46,2 & 0,134 \\
\hline & Não & 139 & 67,8 & 66 & 32,2 & \\
\hline \multirow[t]{2}{*}{ Trabalho de parto prematuro§ } & Sim & 15 & 60 & 10 & 40 & 0,691 \\
\hline & Não & 145 & 66,2 & 74 & 33,8 & \\
\hline \multirow[t]{2}{*}{ Infecção do trato urinário } & Sim & 69 & 66,3 & 35 & 33,7 & 0,966 \\
\hline & Não & 92 & 65,2 & 49 & 34,8 & \\
\hline
\end{tabular}

$\S U m$ caso sem registro nessas intercorrências.

\section{DISCUSSÃO}

Constatou-se no presente estudo que 245 mulheres tiveram pelo menos uma intercorrência durante o pré-natal. Em pesquisa realizada em ambulatório de gravidez de alto risco, essa frequência é ainda maior, uma vez que $91,8 \%$ dos prontuários avaliados indicavam que as mulheres passaram por alguma intercorrência obstétrica na gestação atual ${ }^{(13)}$. Em contrapartida, taxas mais baixas de intercorrências na gestação foram encontradas em outras investigações ${ }^{(14-15)}$. Em um instituto de referência para gravidez de alto risco fetal no Rio de Janeiro, 20,3\% das mulheres apresentaram condições clínicas preexistentes e intercorrências clínicas e 45,2\% tinham alguma doença obstétrica na gravidez atual(14). Já em uma maternidade escola, $42,7 \%$ das gestantes atendidas apresentaram alguma intercorrência obstétrica ${ }^{(15)}$.

Em relação à caracterização das mulheres com intercorrência na gestação, pesquisa destaca que $64 \%$ estavam na faixa etária entre 26 e 35 anos, 56\% das mulheres possuíam ensino fundamental completo, $68 \%$ tinham duas ou mais gestações, e $46 \%$, entre um e três partos ${ }^{(11)}$. Tais dados corroboram os encontrados no presente estudo.

As taxas de intercorrências obstétricas crescem conforme aumenta a idade $65,4 \%$ para mulheres de 40 a 44 anos e 125,9\% para as de 45 a 49 anos) $)^{(16)}$. Tal análise não foi realizada no presente estudo, mas ressalta-se que $32(13,1 \%)$ mulheres se encontravam com idade igual ou superior a 35 anos, a qual configura fator de risco gestacional que permite a realização do pré-natal pela equipe de atenção básica ${ }^{(17)}$. Apesar disso, ressalta-se que tais fatores devem ser verificados durante todo o pré-natal, visto que, por exemplo, o risco de complicações da hipertensão arterial aumenta gradualmente com a idade em mulheres grávidas com idade superior a 35 anos. E os riscos de pré-eclâmpsia e eclâmpsia aumentam na gravidez na adolescência ${ }^{(18)}$.

Em relação à renda familiar, pesquisa apresentou que a maioria das mulheres com alguma intercorrência clínica e/ou obstétrica tinha renda familiar entre um e três salários mínimos $(39,4 \%)^{(14)}$. Esse achado é equivalente ao encontrado no presente estudo, possivelmente pelas semelhanças entre as instituições, ambas de referência para gestação de risco, o que caracteriza o perfil das mulheres atendidas.

Ressalta-se que são considerados como fatores de risco gestacional a baixa escolaridade (menos de cinco anos de estudo regular), a nuliparidade e a multiparidade (cinco ou mais partos) $)^{(17)}$, variáveis essas que se mostraram com baixa frequência nesse estudo.

No que se refere às intercorrências na gestação, as complicações hipertensivas ocorrem em cerca 
de $10 \%$ de todas as gestações e recorrem de $20 \%$ a $50 \%$ nas gestações subsequentes ${ }^{(17)}$. Já a infecção do trato urinário ocorre em $17 \%$ a $20 \%$ das mulheres; a amniorrexe prematura tem incidência entre $3 \%$ e $18,5 \%$ e recorrência de $21 \%$; a diabetes gestacional tem prevalência entre $3 \%$ e $13 \%$ das gestações, sendo o problema metabólico mais comum ${ }^{(17)}$. A taxa de prematuridade varia entre $5 \%$ e $15 \%{ }^{(19)}$. No presente estudo, a frequência das complicações estudadas foi semelhante às apontadas para o Brasil.

Considera-se que um ponto chave para um bom seguimento da gestação é o conhecimento das mulheres sobre a sua condição de saúde e a do bebê. Neste estudo, constatamos que uma parcela das mulheres não tinha conhecimento sobre a intercorrência que estavam vivenciando, e isso pode ser considerado um fator agravante para essas mulheres.

Endossando essa questão, 51,4 \% gestantes de uma maternidade da cidade de São Paulo, submetidas ao rastreamento para Streptococcus B, não possuiam registro em suas carteiras de pré-natal e $43,2 \%$ dela relataram nenhum conhecimento ou não lembrar de terem realizado tal exame ${ }^{(20)}$.

O acompanhamento pré-natal não deve englobar somente os procedimentos clínicos, mas também os educativos, pois estes orientam a mulher sobre a evolução da gestação ${ }^{(21)}$. A gestante não deve aceitar somente o que lhe é oferecido, mas também buscar o que é de seu direito, como o conhecimento adequado sobre seus problemas de saúde durante a gestação ${ }^{(22)}$. As orientações sobre intercorrências no pré-natal são extremamente importantes, visto que se refletirão em cuidados a esse grupo de gestantes no que se refere ao tratamento e também na identificação do agravamento de sua condição de saúde e/ou do feto.

No que diz respeito ao tipo de parto, uma pesquisa de coorte realizada com 500 gestantes com préeclâmpsia grave constatou que $22 \%$ das gestantes tiveram trabalho de parto espontâneo e $28,2 \%$ das gestantes o trabalho de parto foi induzido, enquanto $49,8 \%$ teve uma cesariana eletiva. Entre as que tiveram indução, $67,4 \%$ teve parto vaginal, sendo que a taxa de cesariana total foi de $68,2 \%{ }^{(23)}$. Outra pesquisa realizada com gestantes com diagnóstico de síndrome hipertensiva, a via de parto de 78,4\% delas foi cesárea ${ }^{(24)}$. As taxas de cesariana dessas pesquisas foram maiores que as encontradas nesse estudo.

A decisão sobre a via de parto em mulheres com síndromes hipertensivas deve ser tomada em função da idade gestacional, da vitalidade fetal e da gravidade da doença ${ }^{(24)}$. Em gestantes com préeclampsia/eclâmpsia, o parto vaginal é preferível á cesariana, evitando assim o estresse adicional de um procedimento cirúrgico em situação de alterações fisiológicas múltiplas ${ }^{(3)}$.

Dados de hospital de nível terciário em Portugal, revelou que entre as gestantes com diabetes mellitus gestacional, a taxa de cesariana não eletiva foi de $19,5 \%{ }^{(22)}$. Considera-se importante ressaltar que a diabetes gestacional não é uma indicação absoluta para cesariana. A decisão da via de parto é obstétrica, devendo-se considerar o peso fetal. Caso o peso fetal estimado seja igual ou superior a $4500 \mathrm{~g}$, pode-se considerar a realização de cesariana ${ }^{(3)}$.

Em gestantes com amniorrexe prematura em um hospital universitário, a taxa de cesariana foi $36,9 \%{ }^{(25)}$. Pesquisa realizada não descreve a amniorrexe prematura como indicação para cesárea, devendo, portanto, serem analisados os riscos e benefícios tanto para a mãe quanto para o feto ${ }^{(26)}$. À redução do volume do líquido amniótico, soma-se avaliação da idade gestacional, considerado parâmetro fundamental na tomada de $\operatorname{conduta}^{(3)}$.

Diante do exposto, ressalta-se que o melhor tipo de parto para mulher deve ser aquele mais adequado às suas condições gestacionais e o que melhor atender o seu bebê ${ }^{(17)}$. Assim, durante a gravidez devem ser considerados os fatores de riscos gestacionais, assegurando um bom desenvolvimento e desfecho da gestação e o nascimento de um recém-nascido saudável.

\section{CONCLUSÃO}

Concluímos que 245 mulheres entrevistadas tiveram uma ou mais intercorrências clínicas e/ou obstétricas registradas na carteira de pré-natal, sendo a mais frequente a infecção do trato urinário. Houve maior coincidência entre registro na carteira e relato da mulher nos casos de síndrome hipertensiva, infecção do trato urinário, trabalho de parto prematuro e diabetes gestacional, e um 
número expressivo de mulheres não tinha conhecimento sobre a intercorrência.

Tal situação pode trazer repercussões maiores, como a não-adesão ao tratamento por não saber ou não entender que vem passando por uma situação atípica na gestação, e pelo desconhecimento dos sinais de agravamento de tal intercorrência. Não podemos deixar de considerar que o elevado número de mulheres com alguma intercorrência na gestação pode estar relacionado com a qualidade da atenção pré-natal.

Considera-se que a caracterização do perfil dessas mulheres com intercorrências na gestação é importante, visto que o conhecimento desses dados possibilita o planejamento de ações que possam direcionar medidas de promoção de saúde para esse grupo de mulheres. Dessa forma, a equipe de enfermagem poderá intensificar medidas de educação em saúde e estratégias de prevenção desses acometimentos, norteando a assistência às gestantes atendidas na instituição estudada.

Sugerem-se novas pesquisas direcionadas à temática, no sentido de elucidar os motivos pelos quais as mulheres não têm conhecimento sobre o que está acontecendo durante o período gestacional. As limitações deste estudo estão relacionadas à presença de poucos estudos brasileiros que possam subsidiar maior aprofundamento na temática.

\section{REFERÊNCIAS}

1. Fundo das Nações Unidas para a Infância (UNICEF). Guia dos Direitos da Gestante e do Bebê. [Internet] São Paulo: Globo; 2011 [acesso em 16 nov 2015]. Disponível:

http://www.unicef.org/brazil/pt/br_guiagestantebebe.pdf.

2. Fescina RH, de Mucio B, Díaz Rossello JL, Martínez G, Granzotto JA, Schwarcz RL. Saúde sexual e reprodutiva: guias para a atenção continuada de mulher e do recém-nascido focalizadas na APS. Montevidéu: CLAP/SMR; 2010.

3. Ministério da Saúde (BR). Secretaria de Atenção à Saúde. Departamento de Ações Programáticas Estratégicas. Gestação de alto risco: manual técnico. $5^{\text {a }}$ ed. Brasília: Editora do Ministério da Saúde; 2012.

4. Secretaria de Estado da saúde de São Paulo. . Coordenadoria de planejamento em saúde. Assessoria técnica em saúde da mulher. Atenção à gestante e à puérpera no SUS-SP: manual técnico do pré-natal e puerpério. São Paulo: SES/SP; 2010.

5. Ministério da Saúde (BR). Nascidos vivos - DATASUS [Internet]. Brasília: Departamento de Informática do SUS. Informações de Saúde. Estatísticas Vitais; 2013 [acesso em 16 nov 2015]. Disponível:

http://tabnet.datasus.gov.br/cgi/tabcgi.exe?sinasc/cnv/nvuf.def.

6. Instituto Brasileiro de Geografia e Estatística (IBGE). IBGE divulga indicadores demográficos de saúde. IBGE Sala de Imprensa - Notícias [Internet] Brasília; 2009 [acesso em 16 nov 2015]. Disponível: http://saladeimprensa.ibge.gov.br/noticias?view=noticia\&id=1\&busca=1\&idnoticia=1445.

7. Stein-Backes MT, Flores-Soares MC. Las enfermerdades intercurrentes durante la gestación y sus consecuencias sobre el peso del recién nacido. Rev Colomb Obstet Ginecol. [Internet] 2008; 59(2) [acesso em 16 nov 2015]. Disponível: http://www.scielo.org.co/scielo.php?script=sci_arttext\&pid=S0034-74342008000200004\&lng=en.

8. Ministério da Saúde (BR). Secretaria de Vigilância em Saúde. Departamento de Análise de Situação de Saúde. Saúde Brasil 2013: uma análise da situação de saúde e das doenças transmissíveis relacionadas à pobreza. Brasília: Ministério da Saúde; 2014.

9. Programa das Nações Unidas para o Desenvolvimento (PNUD). Mortalidade materna cai 55\% no Brasil, de acordo com relatório ODM. [Internet] 2014 [acesso em 16 nov 2015]. Disponível:

http://www.pnud.org.br/Noticia.aspx?id=3933.

10. Instituto de Pesquisa Econômica Aplicada (IPEA). Objetivos de desenvolvimento do milênio: relatório nacional de acompanhamento. [Internet] Brasília: IPEA; 2014 [acesso em 16 nov 2015]. Disponível:

http://www.pnud.org.br/Docs/5_RelatorioNacionalAcompanhamentoODM.pdf. 
11. Cabral RWL, Medeiros AL, Pinto LNMR, Caroline P, Durier IS. Atuação do enfermeiro nas intercorrências e complicações obstétricas durante o trabalho de parto e nascimento. In: VII Congresso Brasileiro de Enfermagem Obstétrica e Neonatal. Minas Gerais: ABENFO-MG; 2011.

12. Ministério da Saúde (BR). Conselho Nacional de Saúde. Diretrizes e normas regulamentadoras de pesquisa envolvendo seres humanos. Resolução n. 466, de 12 de dezembro de 2012. Brasília; 2012.

13. Marreiro CM, da Paixão NCF, Brito NMB, Cavalcante JCW. Perfil clínico-epidemiológico das pacientes atendidas no ambulatório de gravidez de alto risco da Fundação Santa Casa de Misericórdia do Pará. Rev. para med. [Internet] 2009; 23(3) [acesso em 16 nov 2015]. Disponível:

http://files.bvs.br/upload/S/0101-5907/2009/v23n3/a1972.pdf.

14. Xavier RB, Jannotti CB, da Silva KS, Martins AC. Risco reprodutivo e renda familiar: análise do perfil das gestantes. Ciênc. saúde coletiva. [Internet] 2013; 18(4) [acesso em 16 nov 2015]. Disponível:

http://dx.doi.org/10.1590/S1413-81232013000400029.

15. Campos LF, Saunders C, Ramalho A, Gomes MM, Accioly E. Níveis de retinol e carotenóides séricos e intercorrências gestacionais em puérperas. Rev. Nutr. [Internet] 2008; 21(6) [acesso em 16 nov 2015]. Disponível: http://dx.doi.org/10.1590/S1415-52732008000600002.

16. Veras TCS, Mathias TAF. Principais causas de internações hospitalares por transtornos maternos. Rev. esc. enferm. USP. [Internet] 2014; 48(3) [acesso em 16 nov 2015]. Disponível:

https://dx.doi.org/10.1590/S0080-623420140000300003.

17. Ministério da Saúde (BR). Secretaria de Atenção à Saúde. Departamento de Atenção Básica. Atenção ao prénatal de baixo risco. [Internet] Brasília: Ministério da Saúde; 2012 [acesso em 16 nov 2015]. Disponível:

http://bvsms.saude.gov.br/bvs/publicacoes/cadernos_atencao_basica_32_prenatal.pdf.

18. Liu X, Ruan Y, Liu Y, Zhang W. Relationship between maternal age and hypertensive disorders in pregnancy. Zhonghua Yi Xue Za Zhi. [Internet] 2015; 95(1) [acesso em 11 mar 2016]. Disponível:

http://www.ncbi.nlm.nih.gov/pubmed/25876802.

19. Rabello MSC, de Barros SMO. Aspectos clínicos e epidemiológicos da prematuridade em um Centro de Parto Normal. Einstein (São Paulo). [Internet] 2011; 9(4) [acesso em 16 nov 2015]. Disponível:

http://apps.einstein.br/revista/arquivos/PDF/1874-Einstein_v9n4_483-488_eng.pdf.

20. de Mello DS, Tsunechiro MA, Mendelski CA, Pierre SA, Silva AR, Padoveze MC. Group B Streptococcus: compliance with the information in prenatal card records and knowledge of pregnant women. Am J Infect Control. [Internet] 2015; 1;43 [acesso em 11 mar 2016] Disponível: http://dx.doi.org/10.1016/j.ajic.2014.12.026.

21. Barreto MS, Mathias TAF. Cuidado à gestante na atenção básica: relato de atividades em estágio curricular. Rev Rene. [Internet] 2013; 14(3) [acesso em 16 nov 2015]. Disponível:

http://www.revistarene.ufc.br/revista/index.php/revista/article/view/1398.

22. Ministério da Saúde (BR). Secretaria Executiva. Programa Humanização do Parto: humanização no pré-natal e nascimento. [Internet] Brasília: Ministério da Saúde; 2002 [acesso em 16 nov 2015]. Disponível:

http://bvsms.saude.gov.br/bvs/publicacoes/parto.pdf.

23. Amorim MM, Katz L, Barros AS, Almeida TS, Souza AS, Faúndes A. Maternal outcomes according to mode of delivery in women with severe preeclampsia: a cohort study. J Matern Fetal Neonatal Med. [Internet] 2015; 28(6) [acesso em 11 mar 2016]. Disponível: http://dx.doi.org/10.3109/14767058.2014.928689.

24. Linhares JJ, Macêdo NMQ, de Arruda GM, Vasconcelos JLM, Saraiva TV, Ribeiro AF. Fatores associados à via de parto em mulheres com pré-eclâmpsia. Rev. Bras. Ginecol. Obstet. [Internet] 2014; $36(6)$ [acesso em 16 nov 2015]. Disponível: https://dx.doi.org/10.1590/S0100-720320140004812.

25. Scandiuzzi MM, Pinheiro TC, Rodrigues AAN, Alcadipani FAMC. Resultados maternos e perinatais na ruptura prematura de membranas. Rev. Fac. Ciênc. Méd. Sorocaba. [Internet] 2014; 16(4) [acesso em 16 nov 2015$].$ Disponível: http://revistas.pucsp.br/index.php/RFCMS/article/view/19290/pdf.

26. Campos MEAL, Alcantara DF, Gois LPBL. Incidência e características de cesáreas e partos normais: um estudo comparativo. 15 CBCENF. [Internet] 2012 [acesso em 16 nov 2015]. Disponível:

http://apps.cofen.gov.br/cbcenf/sistemainscricoes/arquivosTrabalhos/I35081.E10.T6094.D6AP.pdf. 